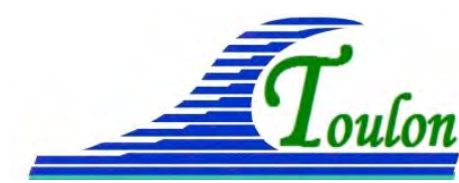

XIV èmes Journées Nationales Génie Côtier - Génie Civil Toulon, 29 juin au $1^{\text {er }}$ juillet 2016

DOI:10.5150/jngcgc.2016.002 (c) Editions Paralia CFL disponible en ligne - http://www.paralia.fr - available online

\title{
Propagation de la houle à travers un milieu poreux : effets 3D
}

\section{Gwendoline ARNAUD ${ }^{1,2}$, Vincent REY ${ }^{1}$, Julien TOUBOUL ${ }^{1}$, Damien SOUS ${ }^{1}$}

1. Université de Toulon, CNRS/INSU, IRD, Mediterranean Institute of Oceanography (MIO), UM 110, 83041 Toulon Cedex 09, France.

Aix Marseille Université, CNRS/INSU, IRD, Mediterranean Institute of

Oceanography (MIO), UM 110, 13288 Marseille, France.

garnaud@univ-tln.fr ; rey@univ-tln.fr ; touboul@univ-tln.fr ; sous@univ-tln.fr

2. ACRI-In, 260, route du Pin Montard Sophia-Antipolis F-06410 Biot, France.

\section{Résumé :}

Lors d'une précédente étude en 2D (ARNAUD et al., 2014), nous avions étudié les caractéristiques d'un milieu poreux constitué d'un réseau dense de cylindres émergeants via les mesures des coefficients de transmission et de réflexion. Nous avions en particulier caractérisé la relation de dispersion à partir du modèle proposée par GU et WANG (1991) dans la limite de milieux non dissipatifs, et avions étudié l'atténuation de l'onde à travers le milieu poreux en supposant un taux de dissipation constant par unité de longueur d'onde. Nous nous intéressons ici au cas de la diffraction d'une onde de gravité par un milieu poreux émergent de forme rectangulaire. Les expériences ont été menées dans le bassin d'essai de SeaTech pour différentes conditions de houles régulières. Elles sont confrontées à un modèle numérique linéaire basé sur la résolution intégrale des conditions de continuité de vitesses et de pression. Les résultats expérimentaux montrent une diffraction de la houle par le milieu poreux. En amont du milieu poreux dans l'axe médian du bassin, l’onde partiellement stationnaire présente un comportement similaire à celui observé lors des études précédentes en 2D. Ces résultats sont en bon accord avec l'approche théorique, qui montre en outre une légère modification de la longueur d'onde au niveau de l'axe médian à cause des effets 3D.

Mots-clés : Houle, Milieu poreux, Dissipation, Diffraction, Réflexion.

\section{Introduction}

Dans le cadre de la protection du littoral, de nombreuses structures sont placées en amont des plages ou des ouvrages portuaires dans le but de réfléchir et d'atténuer l'énergie de la houle incidente. Les ouvrages classiques de type milieu poreux (notamment enrochements, tétrapodes, etc..) font l'objet de nombreuses études, pour mieux comprendre et modéliser l'hydrodynamique à travers ce type de milieu, et son impact sur les caractéristiques de la houle dans son champ proche.

Certaines de ces études, menées principalement en 2D, ont mis en évidence l'importance des effets inertiels et non-linéaires, pris en compte dans une relation de 


\section{Thème 1 - Hydrodynamique côtière}

dispersion complexe, sur l'atténuation de la houle (GU \& WANG, 1991 et références citées). Les phénomènes tels que la réflexion et l'atténuation de la houle par des structures poreuses ont été également quantifiés expérimentalement (SOLLITT \& CROSS, 1972). YU et CHWANG (1994) ont étudié l'impact de blocs poreux superposés sur la propagation de la houle, pour des milieux faiblement à fortement dissipatifs. Les modes solutions de la relation de dispersion proposée, complexe, faisaient donc apparaitre un terme de propagation et un terme de dissipation lié aux caractéristiques de l'onde. Dans notre étude, le milieu poreux est composé de cylindres denses émergeants. Lors de nos précédentes études en 2D (ARNAUD et al., 2014), nous avons opté pour une formulation un peu différente, avec une relation de dispersion réelle, issue de la formulation proposée par YU et CHWANG (1994), mais dans la limite d'un milieu non dissipatif. Cette approche était motivée par une longueur caractéristique de la dissipation très supérieure à l'échelle des cylindres. Nos calculs étaient basés sur une formulation linéaire de la dissipation. En outre, l'absence de discontinuité de profondeur d'eau aux frontières verticales du milieu poreux nous a permis de négliger les modes évanescents dans notre formulation (nos calculs ont, en effet, montré que leur poids était négligeable dans le processus d’interférences).

Les premiers résultats théoriques concernant la diffraction de vagues linéaires par un réseau de cylindres émergeants ont été obtenus en utilisant la théorie des fonctions propres (SPRING \& MONKMEYER, 1974). Ces résultats théoriques ont ensuite été simplifiés par LINTON et EVANS (1990). En parallèle, d'autres théories ont été développées comme celle de KAGEMOTO et YUE (1986). La diffraction par un ensemble d'éléments est alors résolue de façon exacte en trois dimensions. Sur la base de ces études, WILLIAMS et LI (2000) se sont intéressés théoriquement à la propagation de la houle au voisinage d'un réseau de cylindres poreux. De plus, YU (1995) a décrit une nouvelle solution dérivée pour la diffraction des vagues par de fines digues poreuses à l'aide d'une condition limite à l'interface du milieu poreux résolue en deux dimensions. L’approche présentée ici considère le réseau de cylindres émergents comme un milieu poreux homogène à l'échelle de l'onde. Des expressions générales des potentiels des vitesses pour chacun des milieux finis ou semi-infinis constituant le milieu de propagation de l'onde sont ainsi définies. La solution au problème est obtenue en résolvant sous forme intégrale les conditions de continuité de vitesse et de pression aux frontières verticales entre les domaines. Après une présentation du dispositif expérimental et du modèle théorique, les résultats sont discutés.

\section{Dispositif expérimental}

\subsection{Bassin d'essai et milieu poreux}

Le bassin d'essai de SeaTech, Université de Toulon, possède une longueur utile de $10 \mathrm{~m}$ et une largeur efficace de $2.60 \mathrm{~m}$ (voir figure 1). La hauteur d'eau maximale est de $1 \mathrm{~m}$. 


\section{XIV $V^{\text {èmes }}$ Journées Nationales Génie Côtier - Génie Civil \\ Toulon, 29 juin au $1^{\text {er }}$ juillet 2016}

Le batteur à houle permet de créer d'une part des houles régulières de période de 0.5 - $2 \mathrm{~s}$ et d'autre part des houles irrégulières.

Le milieu poreux "modèle" est constitué d'un réseau dense de cylindres verticaux émergeants, disposés régulièrement selon deux axes perpendiculaires faisant un angle de $45^{\circ}$ avec l'axe longitudinal du canal. La structure poreuse est de longueur $\mathrm{L}=0.30 \mathrm{~m}$ et largeur $2 \mathrm{~d}_{\mathrm{p}}=1.20 \mathrm{~m}$. Le diamètre des cylindres $\mathrm{D}$ est égal $0.032 \mathrm{~m}$. La porosité $\gamma \mathrm{du}$ milieu poreux est égale à 0.7. La hauteur d'eau pour la série de mesures est constante et égale à $0.23 \mathrm{~m}$, via un faux fond disposé dans le bassin. Elle est donc identique à celle relative aux expériences précédentes menées en 2D (ARNAUD et al., 2014).

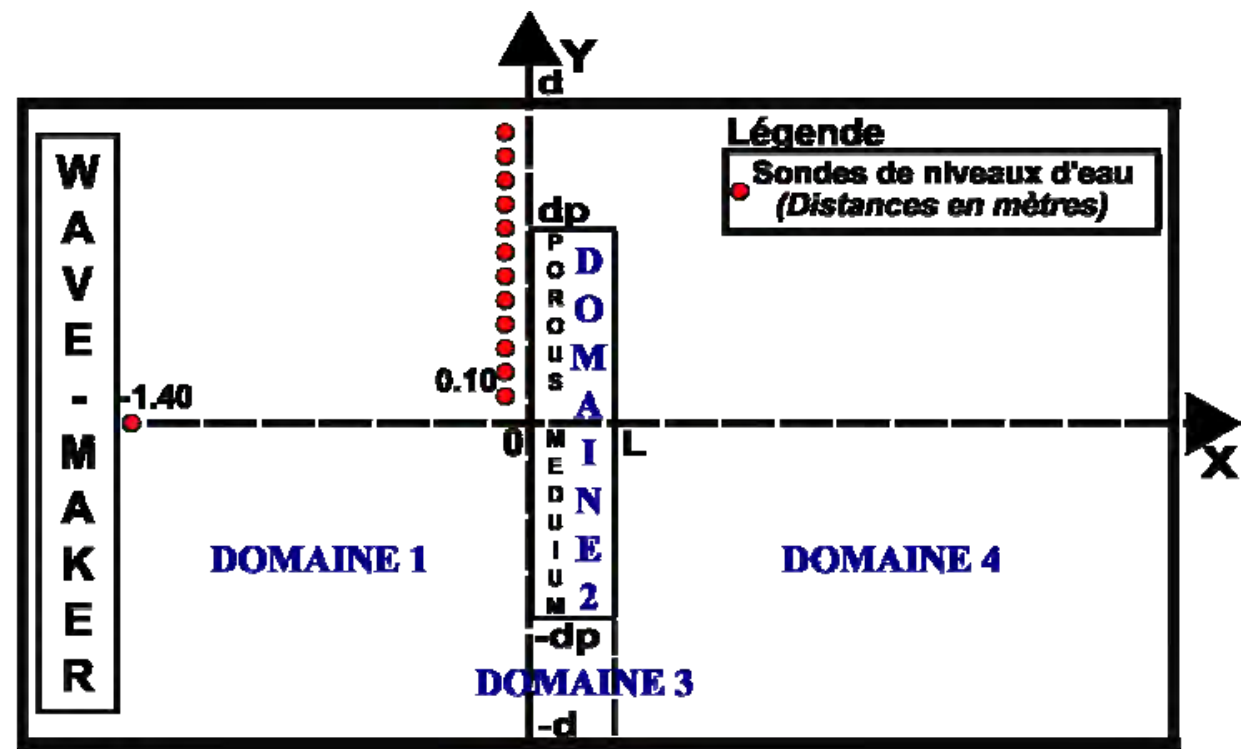

Figure 1. Schéma du dispositif expérimental.

\subsection{Conditions d'expériences et instrumentation}

Cette série d'expériences avait pour but de cartographier l'ensemble de la surface libre autour du milieu poreux. La surface libre était mesurée à l'aide de 13 sondes acoustiques synchrones, de fréquence d'échantillonnage $40 \mathrm{~Hz}$. La cartographie a été réalisée selon une grille régulière de 0.10 par $0.10 \mathrm{~m}$. En effet, les sondes étaient séparées de $0.10 \mathrm{~m}$ et la ligne a été déplacée de $0.10 \mathrm{~m}$ entre chaque mesure. Huit conditions de houle (voir tableau 1) ont été réalisées.

Tableau 1. Synthèse des conditions de houle pour la cartographie de la surface libre dans le bassin d'essai.

\begin{tabular}{l|lll}
\hline Fréquences & \multicolumn{3}{|c}{ Amplitudes } \\
\hline 1 & 0.010 & 0.015 & 0.020 \\
1.5 & 0.010 & 0.015 & 0.020 \\
1.8 & 0.010 & 0.015 & \\
\hline
\end{tabular}




\section{Modèle théorique}

L'amplitude de la houle est supposée faible, l'écoulement du fluide dans le régime linéaire dérive d'un potentiel des vitesses. On considère un repère cartésien $(\mathrm{O}, \mathrm{x}, \mathrm{y}, \mathrm{z})$, l'axe Ox correspondant à la direction de propagation de la houle incidente, Oz étant l'axe vertical orienté vers le haut. La surface libre est donnée par $\mathrm{z}=0$, l'origine des axes correspond au milieu (suivant Oy) du bord d'attaque de la structure (voir figure 1). Par symétrie du problème, on se place dans le demi-espace $\mathrm{y}>0$. Pour un domaine $\mathrm{j}$ de profondeur constante $h$, de largeur finie, délimité par $\mathrm{d}_{\mathrm{jm}}<\mathrm{y}<\mathrm{d}_{\mathrm{jM}}$, l'expression générale du potentiel des vitesses $\Phi_{j}, j=1, . .4$, en l'absence de dissipation est de la forme :

$\Phi_{j}(x, y, z, t)=\cosh \left[k_{j}(z+h)\right] \sum_{n=0}^{\infty}\left[A_{j n}^{-} e^{-i k_{j x n} x}+A_{j n}^{+} e^{+i k_{j x n}(x-L)}\right] \cos \left[k_{j y n}\left(y-d_{j m}\right)\right] e^{i \omega t}$

où $k_{j y n}=\frac{n \pi}{d_{j M}-d_{j m}}$.

Comme $\nabla^{2} \Phi_{j}=0, k_{j x n}=\sqrt{\left(k_{j}^{2}-k_{j y n}^{2}\right)}$. Le vecteur d'onde $k_{j}$ est donné par la relation de dispersion suivante :

$\omega^{2} S=g k_{j} \tanh \left(k_{j} h\right)$

où $S$ est la réactance, définie par $S=1+C_{m}(\gamma-1) / \gamma \cdot C_{m}$ est un coefficient de masse ajoutée égal à 0.3 pour le domaine poreux (ARNAUD et al., 2014), et est nul pour les trois autres domaines non poreux. Le premier mode, $n=0$, correspond à une onde se propageant dans la direction Ox. Pour $\mathrm{n}>0$, l'onde se propage dans une direction faisant un angle $\theta_{j n}=\arctan \left(k_{j y n} / k_{j x n}\right)$ si $k_{j y n}<k_{j n}$. Au-delà d'une certaine valeur $\mathrm{n}_{\mathrm{jp}}$ de n, les modes deviennent évanescents dans la direction Ox. Pour le domaine 1, seule l'onde incidente se propage vers les $\mathrm{x}>0$ et les modes évanescents doivent rester finis quand $x \rightarrow-\infty$. Le coefficient $A^{-} 11$, correspondant à l'onde incidente est connu, $A^{-}{ }_{1 n}=0$ pour tout $\mathrm{n}>0$, et $k_{1 x n}=i \sqrt{k_{1}^{2}-k_{1 y n}^{2} \mid}$ pour $\mathrm{n}>\mathrm{n}_{1 \mathrm{p}}$. Pour le domaine 4 , en l'absence de réflexion de la plage, $A^{+}{ }_{4 n}=0$ pour tout $\mathrm{n}$, et $k_{1 \times n}=-i \sqrt{\left|k_{4}^{2}-k_{4 y n}^{2}\right|}$ pour $\mathrm{n}>\mathrm{n}_{4 \mathrm{p}}$. Le nombre de coefficients inconnus est donc $6(\mathrm{~N}+1)$ en se limitant aux $\mathrm{N}+1$ premiers modes. L'atténuation dans le milieu poreux est prise en compte en écrivant pour les modes propagatifs $\mathrm{k}=\mathrm{k}_{\mathrm{p}}+\mathrm{i} \mathrm{k}_{\mathrm{d}}$ avec $\mathrm{k}_{\mathrm{d}}=\mathrm{k}_{\mathrm{p}} / \mathrm{n}_{\text {diss }}\left(\mathrm{n}_{\text {diss }}=20\right.$ dans cette étude), c'est-à-dire en considérant que le taux d'atténuation de l'onde est de $e^{-2 \pi / n_{\text {diss }}}$ par unité de longueur d'onde.

La longueur du milieu poreux étant très inférieure aux longueurs d'onde dans notre cas d'étude, les flux à travers les parois latérales du milieu poreux ont été négligés. Les conditions de continuités des vitesses et des pressions aux interfaces $\mathrm{x}=0$ (respectivement $\mathrm{x}=\mathrm{L}$ ) imposent :

$$
\phi_{i}=\gamma \phi_{j} \quad \text { et } \quad \partial \phi_{i} / \partial x=S \partial \phi j / \partial x \quad \text { pour } \quad 0<\mathrm{y}<\mathrm{d}_{\mathrm{p},}-\mathrm{h}<\mathrm{z}<0
$$

avec $i$ l'indice correspondant au domaine 1 (respectivement domaine 3), $j$ l'indice du domaine 2 (milieu poreux) et 


\section{XIV èmes Journées Nationales Génie Côtier - Génie Civil \\ Toulon, 29 juin au $1^{\text {er }}$ juillet 2016}

$\phi_{i}=\phi_{j} \quad$ et $\quad \partial \phi_{i} / \partial x=\partial \phi j / \partial x \quad$ pour $\quad \mathrm{d}_{\mathrm{p}}<\mathrm{y}<\mathrm{d},-\mathrm{h}<\mathrm{z}<0$

avec $i$ l'indice correspondant au domaine 1 (respectivement domaine 3), $j$ l'indice du domaine 3.

Ces conditions sont écrites sous forme intégrale, en utilisant comme fonctions propres les fonctions $\cos \left(k_{y n} y\right)$ du domaine 1 (ou 3).

Les conditions de continuité de pression en $\mathrm{x}=0$ (respectivement en $\mathrm{x}=\mathrm{L}$ en remplaçant l'indice 1 par l'indice 3) sont les suivantes :

$$
\begin{aligned}
& \int_{z=-h}^{0} \int_{y=0}^{d_{p}} \Phi_{1} \cos \left(k_{1 y n} y\right) d y d z=\int_{z=-h}^{0} \int_{y=0}^{d_{p}} \Phi_{2} \cos \left(k_{1 y n} y\right) d y d z, n=0, \ldots, N \\
& \int_{z=-h}^{0} \int_{y=0}^{d_{p}} \Phi_{1} \cos \left(k_{1 y n} y\right) d y d z=\int_{z=-h}^{0} \int_{y=d_{p}}^{d} \Phi_{3} \cos \left(k_{1 y n} y\right) d y d z, n=0, \ldots, N
\end{aligned}
$$

Les conditions de continuité de flux en $x=0$ (respectivement en $x=L$ en remplaçant l'indice 1 par l'indice 3) sont intégrées sur toute la largeur du bassin, elles sont données par :

$$
\begin{aligned}
& \int_{z=-h}^{0} \int_{y=0}^{d_{p}} \frac{\partial \Phi_{1}}{\partial x} \cos \left(k_{1 y n} y\right) d y d z=\int_{z=-h}^{0} \int_{y=0}^{d p} \frac{\partial \Phi_{2}}{\partial x} \cos \left(k_{1 y n} y\right) d y d z \\
& +\int_{z=-h}^{0} \int_{y=d_{p}}^{d} \frac{\partial \Phi_{3}}{\partial x} \cos \left(k_{1 y n} y\right) d y d z, n=0, \ldots, N
\end{aligned}
$$

Les $6(\mathrm{~N}+1)$ coefficients inconnus sont alors calculés par résolution de ces $6(\mathrm{~N}+1)$ équations complexes. En l'absence de dissipation, le flux d'énergie de la houle dans la direction Ox, intégré sur la largeur d du bassin, est conservé :

$$
\left|A^{-}{ }_{11}\right|^{2}=\left|A^{+}{ }_{11}\right|^{2}+\left|A^{-}{ }_{41}\right|^{2}+1 / 2 \sum_{n=1}^{n_{p}}\left[\left|A^{+}{ }_{1 n}\right|^{2}+\left|A^{-}{ }_{4 n}\right|^{2}\right]
$$

\section{Résultats expérimentaux et discussions}

\subsection{Champs de hauteurs}

Les figures 2 et 3 représentent les champs des amplitudes de la houle autour du milieu poreux, obtenues expérimentalement et théoriquement pour $\mathrm{f}=1 \mathrm{~Hz}$. La figure 4 représente le motif des amplitudes de houle dans le cas où le milieu poreux serait remplacé par une structure imperméable. Ces résultats montrent ainsi des amplitudes de houle plus importantes en amont de la structure lorsqu'elle est imperméable, la réflexion de la houle est d'autant plus importante. En aval de la structure imperméable la houle est donc très peu transmise, ce qui se traduit par des amplitudes de houle plus faible que dans le cas du milieu poreux. Les figures 6 et 7 représentent les champs d'amplitudes pour $\mathrm{f}=1.8 \mathrm{~Hz}$. Les résultats expérimentaux et numériques font apparaitre une diffraction significative de la houle autour de la structure poreuse, matérialisée en blanc sur les figures. En amont de la structure, une succession de maxima et de minima 


\section{Thème 1 - Hydrodynamique côtière}

d'amplitude sont observés, correspondant à une réflexion partielle de la structure poreuse. Le long de la direction Oy, des modulations sont observées en amont, ce qui peut correspondre à l'excitation de modes transverses résonants. En effet, les longueurs d'onde de l'onde sont respectivement de $1.27 \mathrm{~m}$ et $0.48 \mathrm{~m}$ pour les fréquences $\mathrm{f}=1 \mathrm{~Hz}$ et $\mathrm{f}=1.8 \mathrm{~Hz}$, la largeur du bassin est de $2.60 \mathrm{~m}$, soit des conditions proches de la résonance, notamment pour $\mathrm{f}=1 \mathrm{~Hz}$.

\subsection{Hauteurs de houle près de l'axe médian}

Une coupe horizontale de l'amplitude est réalisée en $\mathrm{Y}=0.10 \mathrm{~m}$, position voisine de l'axe médian du bassin. Ce transect est matérialisé sur les figures 2 et 6 , les hauteurs de houle, mesurées et calculées, sont représentées sur les figures 5 et 9 , respectivement pour $\mathrm{f}=1 \mathrm{~Hz}$ et $\mathrm{f}=1.8 \mathrm{~Hz}$. L'amplitude calculée dans le cas 2D (structure de largeur infinie) est également présentée.

Pour $\mathrm{f}=1 \mathrm{~Hz}$, on observe un très bon accord entre mesures et calculs en amont de la structure poreuse. Les amplitudes théoriques restent assez proches de celles calculées en 2D, avec toutefois un léger décalage des maxima et une légère irrégularité des extrema dans le cas 3D à cause des effets de diffraction. En aval de la structure, les mesures font apparaitre des amplitudes plus élevées que la théorie, avec en particulier des maxima nettement plus prononcés et décalés dans l'espace. Dans le cas 2D, une onde progressive est observée en aval avec une amplitude moins élevée que l'amplitude mesurée. Les principales différences en aval de la structure s'expliquent par les réflexions parasites sur les bords du bassin d'essai. Ces derniers résultats peuvent aussi être visualisés sur les figures expérimentales de réfraction-diffraction (figure 2 et figure 6) où des zones de fortes amplitudes apparaissent sur les bords du bassin.

Pour $\mathrm{f}=1.8 \mathrm{~Hz}$, on observe encore un accord correct entre mesures et calculs en amont de la structure poreuse même si une meilleure résolution spatiale aurait permis une analyse plus précise. En aval de la structure, les mesures font apparaitre des amplitudes plus élevées que la théorie, avec des maxima nettement moins marquées que ceux prévus par la théorie. Dans le cas 2D, l'onde progressive en aval de la structure est en accord avec les données expérimentales.

\section{Discussions et perspectives}

Les expériences menées dans le bassin d'essai de SeaTech ont permis d'appréhender l'impact d'une structure poreuse de dimensions fines sur la propagation d'une houle régulière. Les résultats expérimentaux ont permis d'observer le champ 3D des hauteurs de vagues, ainsi que leurs phases (non présentées ici). Un bon accord entre le modèle théorique et les données expérimentales est observé. Ils montrent aussi la présence de maxima d'amplitude en certains points qui apparaissent importants. L'agitation croissante du bassin au cours du déroulement des expériences, du fait de réflexions multiples sur les parois peut en être une explication. En ce qui concerne le modèle 


\section{XIV èmes Journées Nationales Génie Côtier - Génie Civil \\ Toulon, 29 juin au $1^{\text {er }}$ juillet 2016}

potentiel utilisé, il suppose dans sa version actuelle que le flux est négligeable au niveau des extrémités (suivant y) de la structure. Des travaux sont en cours pour prendre en compte ce flux, qui ne pourra plus être négligé si on considère des structures de longueur significative le long de l'axe de propagation de la houle.

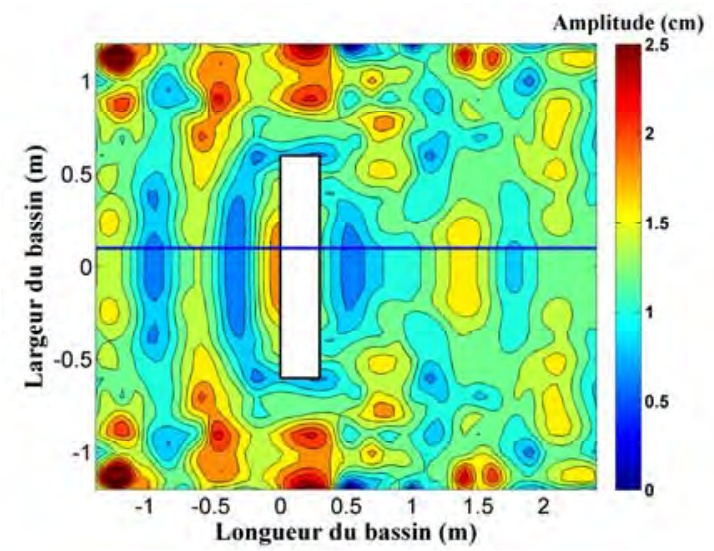

Figure 2. Amplitude mesurée pour $\mathrm{f}=1 \mathrm{~Hz}$ et $a=1 \mathrm{~cm}$.

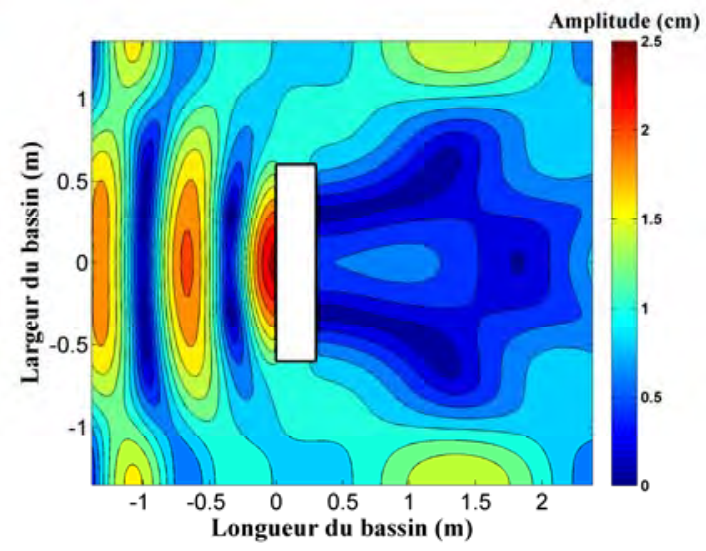

Figure 4. Amplitude théorique pour $f=1 \mathrm{~Hz}$ dans le cas d'une structure imperméable.

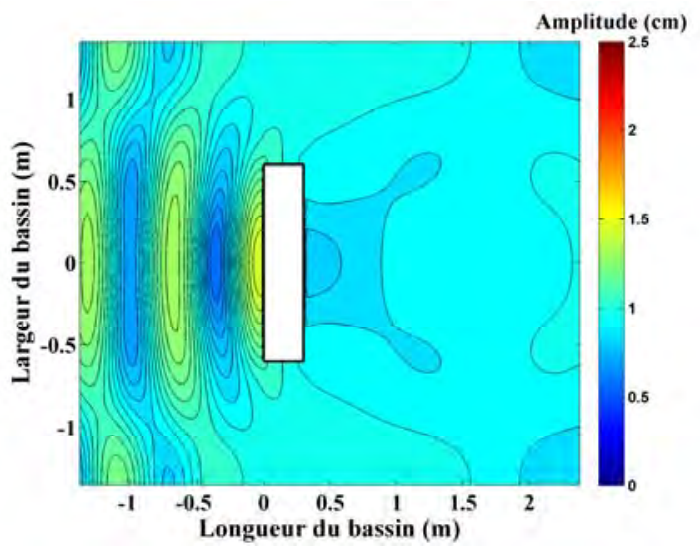

Figure 3. Amplitude théorique pour $f=1 \mathrm{~Hz}$

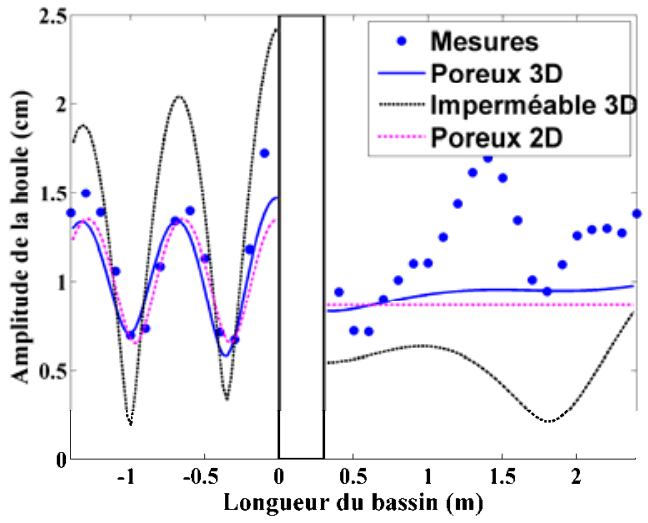

Figure 5. Coupe verticale proche de l'axe $d u \operatorname{bassin}(Y=0.10 \mathrm{~m})$ pour $\mathrm{f}=1 \mathrm{~Hz}$. 


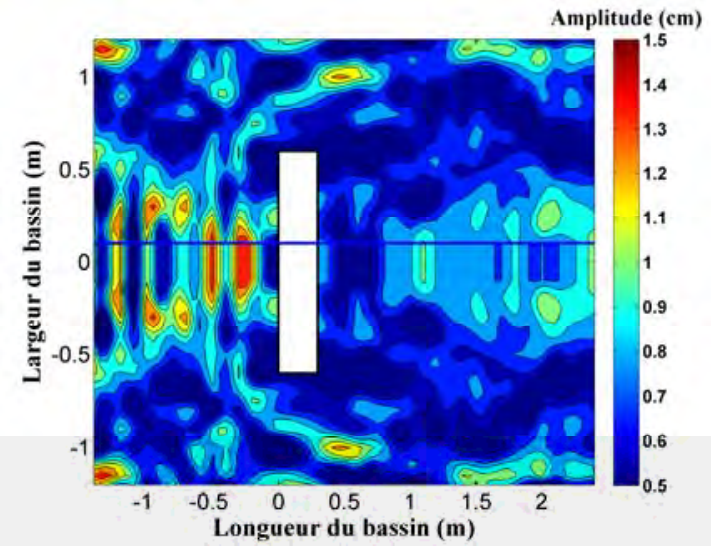

Figure 6. Amplitude mesurée pour

$f=1.8 \mathrm{~Hz}$ et $a=1.5 \mathrm{~cm}$

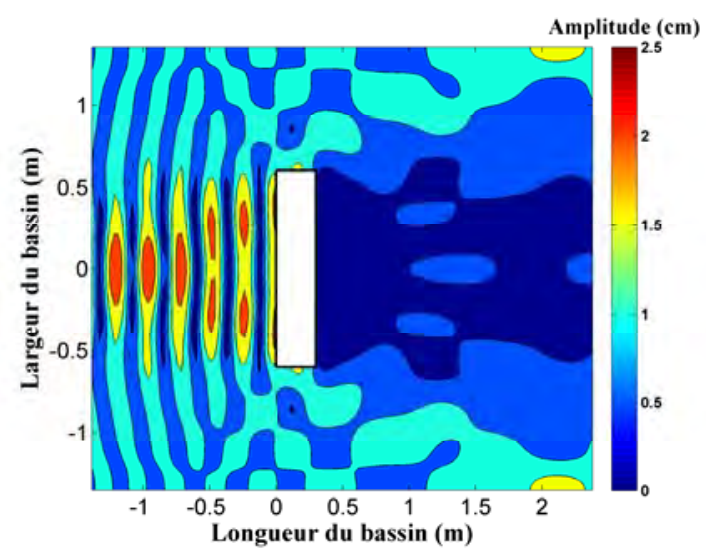

Figure 8. Amplitude théorique pour $f=1.8 \mathrm{~Hz}$ dans le cas d'une structure imperméable.

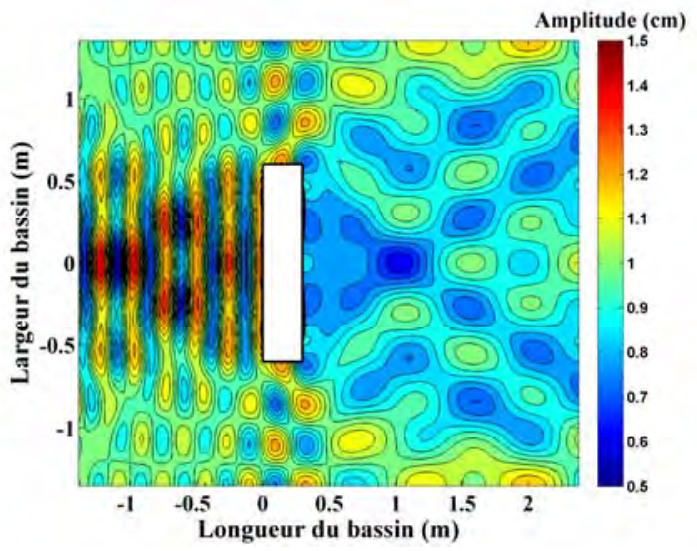

Figure 7. Amplitude théorique pour $f=1.8 \mathrm{~Hz}$.

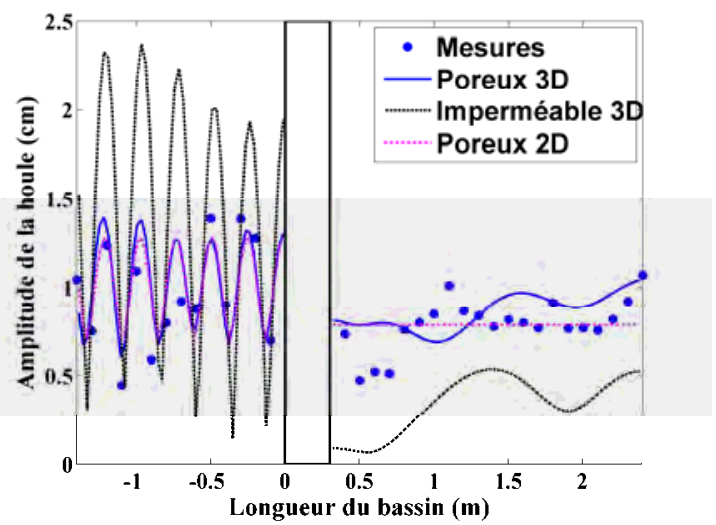

Figure 9. Coupe horizontale proche de l'axe du bassin $(Y=0.10 \mathrm{~m})$ pour $f=1.8 \mathrm{~Hz}$.

\section{Remerciements}

Les auteurs remercient la région "Provence Alpes Côtes-d'Azur" et la société "Acri-In" pour le support financier de la thèse de G. Arnaud. Nos sincères remerciements sont adressés aux rapporteurs de cet article dont les remarques ont permis une amélioration significative des résultats présentés dans cette étude.

\section{Références}

ARNAUD G., TOUBOUL J., SOUS D., GOUAUD F., REY V. (2014). Ecoulements stationnaires et oscillants à travers des milieux poreux : effets de la surface spécifique. Revue Paralia, Vol. 7, pp s04.1-s04.12. http://dx.doi.org/10.5150/revue-paralia.2014.s04 GU Z., WANG H. (1991). Gravity waves over porous bottoms. Coastal Engineering, Vol. 15(5), pp 497-524. http://dx.doi.org/10.1016/0378-3839(91)90025-C 


\section{V̀mes Journées Nationales Génie Côtier - Génie Civil Toulon, 29 juin au $1^{\text {er }}$ juillet 2016}

KAGEMOTO H., YUE D. (1986). Interactions among multiple three-dimensional bodies in water waves: an exact algebraic method. Journal of Fluid Mechanics, Vol. 166, pp 189-209. http://dx.doi.org/10.1017/S0022112086000101

LINTON C., EVANS D. (1990). The interaction of waves with arrays of vertical circular cylinders. Journal of Fluid Mechanics, Vol. 215, pp 549-569. http://dx.doi.org/10.1017/S0022112090002750

SOLLITT C., CROSS R. (1972). Wave transmission through permeable breakwaters. Proceedings of 13th Conference on Coastal Engineering, pp 1827-1846. http://dx.doi.org/10.1061/9780872620490.106

SPRING B.H., MONKMEYER P.L. (1974). Interaction of plane waves with vertical cylinders. Proceedings of 14th Conference on Coastal Engineering, pp 1828-1847. http://dx.doi.org/10.1061/9780872621138.110

WILLIAMS A.N., LI W. (2000). Water wave interaction with an array of bottommounted surface-piercing porous cylinders. Ocean Engineering, Vol. 27(8), pp 841866. http://dx.doi.org/10.1016/S0029-8018(99)00004-9

YU X. (1995). Diffraction of Water Waves by Porous Breakwaters. Journal of Waterway, Port, Coastal, and Ocean Engineering, Vol. 121(6), pp 275-282. http://dx.doi.org/10.1061/(ASCE)0733-950X(1995)121:6(275)

YU X., CHWANG A.T. (1994). Wave motion through porous structures. Journal of Engineering Mechanics, Vol. 120(5), pp 989-1008. http://dx.doi.org/10.1061/(ASCE)07339399(1994)120:5(989) 
Thème 1 - Hydrodynamique côtière 